\title{
Brief cognitive assessment instruments in schizophrenia and bipolar patients, and healthy control subjects: A comparison study between the Brief Cognitive Assessment Tool for Schizophrenia (B-CATS) and the Screen for Cognitive Impairment in Psychiatry (SCIP)
}

\author{
Manuel J. Cuesta ${ }^{\mathrm{a}, *}$, Oscar Pino ${ }^{\mathrm{b}}$, Georgina Guilera ${ }^{\mathrm{c}}$, J. Emilio Rojo ${ }^{\mathrm{b}}$, Juana Gómez-Benito ${ }^{\mathrm{c}, \mathrm{d}}$, \\ Scot E. Purdon ${ }^{\mathrm{e}}$, Manuel Franco ${ }^{\mathrm{f}}$, Anabel Martínez-Arán ${ }^{\mathrm{g}}$, Nuria Segarra ${ }^{\mathrm{h}}$, Rafael Tabarés-Seisdedos ${ }^{\mathrm{i}}$, \\ Eduard Vieta ${ }^{g}$, Miguel Bernardo ${ }^{h}$, Benedicto Crespo-Facorro ${ }^{\mathrm{j}}$, Francisco Mesa ${ }^{\mathrm{k}}$, Javier Rejas ${ }^{1}$ \\ a Psychiatric Hospitalization Unit, Hospital Virgen del Camino, Pamplona-Iruña, Spain \\ b Department of Psychiatry, Benito Menni CASM, Granollers Hospital General, Granollers, Barcelona, Spain \\ c Department of Methodology, Faculty of Psychology, University of Barcelona, Barcelona, Spain \\ d Institute for Brain, Cognition and Behavior (IR3C), Barcelona, Spain \\ e Department of Psychiatry, University of Alberta, Edmonton, Alberta, Canada \\ ${ }^{\mathrm{f}}$ Department of Psychiatry, Hospital Provincial Rodríguez Chamorro, Zamora, Spain \\ g Bipolar Disorders Program, Institute of Neuroscience, Hospital Clinic I Provincial, University of Barcelona, IDIBAPS, CIBERSAM, Barcelona, Spain \\ h Schizophrenia Clinic Program, Institute of Neuroscience, Hospital Clinic I Provincial, University of Barcelona, IDIBAPS, CIBERSAM, Barcelona, Spain \\ i Teaching Unit of Psychiatry and Psychological Medicine, Department of Medicine, University of Valencia, CIBERSAM, Valencia, Spain \\ j Department of Psychiatry, University of Cantabria-IFIMAV, CIBERSAM, Santander 39008, Spain \\ ${ }^{\mathrm{k}}$ Department of Neurosciences, Medical Unit, Pfizer Spain, Alcobendas, Madrid, Spain \\ ${ }^{1}$ Health Outcomes Research Department, Medical Unit, Pfizer Spain, Alcobendas, Madrid, Spain
}

\section{A R T I C L E I N F O}

\section{Article history:}

Received 7 March 2011

Received in revised form 20 May 2011

Accepted 23 May 2011

Available online 11 June 2011

\section{Keywords:}

Cognitive disorders

Cognitive function

Severe mental disorders

Psychiatric endophenotypes

Brief cognitive assessment

\begin{abstract}
A B S T R A C T
Cognitive impairment in schizophrenia and psychosis is ubiquitous and acknowledged as a core feature of clinical expression, pathophysiology, and prediction of functioning. However, assessment of cognitive functioning is excessively time-consuming in routine practice, and brief cognitive instruments specific to psychosis would be of value. Two screening tools have recently been created to address this issue, i.e., the Brief Cognitive Assessment Tool for Schizophrenia (B-CATS) and the Screen for Cognitive Impairment in Psychiatry (SCIP). The aim of this research was to examine the comparative validity of these two brief instruments in relation to a global cognitive score. 161 patients with psychosis (96 patients diagnosed with schizophrenia and 65 patients diagnosed with bipolar disorder) and 76 healthy control subjects were tested with both instruments to examine their concurrent validity relative to a more comprehensive neuropsychological assessment battery. Scores from the B-CATS and the SCIP were highly correlated in the three diagnostic groups, and both scales showed good to excellent concurrent validity relative to a Global Cognitive Composite Score (GCCS) derived from the more comprehensive examination. The SCIP-S showed better predictive value of global cognitive impairment than the B-CATS. Partial and semi-partial correlations showed slightly higher percentages of both shared and unique variance between the SCIP-S and the GCCS than between the B-CATS and the GCCS. Brief instruments for assessing cognition in schizophrenia and bipolar disorders, such as the SCIP-S and B-CATS, seem to be reliable and promising tools for use in routine clinical practice.
\end{abstract}

(c) 2011 Elsevier B.V. All rights reserved.

\footnotetext{
* Corresponding author at: Unidad de Hospitalización Psiquiátrica, Hospital Virgen del Camino, Irunlarrea, 4, 31008-Pamplona-Iruña, Spain. Tel.: +34 848 429400; fax: +34848429924 .

E-mail address: mj.cuesta.zorita@cfnavarra.es (M.J. Cuesta).
}

\section{Introduction}

Cognitive impairment in schizophrenia and bipolar disorder is ubiquitous and recognized as fundamental to both the pathophysiology of these disorders and the prediction of activities of daily living and community functioning (Green et al., 2004, 2005; Tabarés-Seisdedos et al., 2008). There is growing evidence that cognitive impairments are not only detectable before illness onset and stable over time but are also 
shared by first-degree relatives and heritable irrespective of psychopathological diagnosis. Therefore cognitive impairments are among the most promising candidate endophenotypes (Heinrichs and Zakzanis, 1998; Toulopoulou et al., 2007) either of psychosis or its premorbid stages, such as early or transitional phase of psychosis (Heinrichs and Zakzanis, 1998; Brewer et al., 2005).

Neuropsychological deficits are highly prevalent in schizophrenia and bipolar disorder (Daban et al., 2006; Krabbendam et al., 2005; Seidman et al., 2010) and other psychoses, such as schizoaffective disorder (Reichenberg et al., 2009). Although there is still considerable debate regarding the extent to which cognitive deficits and psychopathological symptoms are related, recent reviews and meta-analyses suggest that cognitive impairment and symptoms in schizophrenia are independent or semi-independent dimensions of the illness, since only small to medium significant statistical associations between negative and disorganization dimensions of psychosis and cognitive impairment have been found (Dominguez et al., 2009; Nieuwenstein et al., 2001; Ventura et al., 2000).

Until recently, schizophrenia patients were evaluated by means of extensive neuropsychological batteries specifically designed for neuropsychiatric disorders. There were few brief, valid, and reliable instruments for assessing cognitive functioning by non-trained clinicians in clinical practice. At present, there are specific neuropsychological batteries available for schizophrenia and other psychoses, such as the Cognistat (Kiernan et al., 1987), before 1995 known as the Neurobehavioral Cognitive Status Examination; the Repeatable Battery for the Assessment of Neuropsychological Status (RBANS) (Randolph et al., 1998); the Woodcock-Johnson III Test of Cognitive Abilities (Woodcock et al., 2001); and the Brief Assessment of Cognition in Schizophrenia (BACS) (Keefe et al., 2004; Segarra et al., 2010). Despite the fact that these instruments have excellent psychometric properties, their administration is quite time-consuming (more than $30 \mathrm{~min}$ ), diminishing its utility in psychiatric practice. More abbreviated cognitive assessment instruments could offer an important screening tool for cognitive deficits in psychiatric illnesses, and potentially encourage the monitoring of change over time and treatment. This subject was addressed by clinicians working with dementia and other neuropsychiatric and neurological illnesses years ago with the introduction of standard brief measures, such as the Mini-Mental State Examination (Folstein et al., 1975), now widely used and well validated. In recent years, various brief screening tools have been devised to assess cognition in psychiatric samples with the intention of reducing administration time and bringing it into line with the demands of a typical clinical practice. Examples are the Brief Cognitive Assessment (BCA) (Velligan et al., 2004), the Screen for Cognitive Impairment in Psychiatry (SCIP) (Purdon, 2005); and the Brief Cognitive Assessment Tool for Schizophrenia (B-CATS) (Hurford et al., 2009). These all are simple and easy-to-administer instruments that require minimal additional equipment and have shown good psychometric properties (Guilera et al., 2009; Hurford et al., 2009; Pino et al., 2008; Purdon, 2005; Rojo et al., 2010; Velligan et al., 2004). Until their advent, there were no such instruments designed for detecting cognitive deficits in psychiatric disorders in only 15 min per patient.

As shown in Table 1, both BCA and B-CATS are made up of elements from other tests (e.g., Trail Making Test), while the SCIP is an independent instrument. The BCA, B-CATS, and SCIP share the assessment of key domains of cognitive impairment in psychiatric illnesses. The SCIP and the BCA have subtests of processing speed and attention, verbal memory and learning, verbal fluency, and executive functioning and set-shifting. The B-CATS assesses all these cognitive domains with the exception of verbal memory and learning.

The development of brief instruments should include the study of their comparative validity to reliably summarize and concisely communicate information about a patient's overall level of cognitive functioning. Despite its unquestionable value, the BCA had not generated further studies after its initial publication, and its impact on clinical practice and specialized literature was low. However, both the CATS (and by extension its short version, the B-CATS) and the SCIP are two instruments that seem to be well accepted among clinicians and the scientific community, with a promising future. In this context, the aim of the current study was to examine for the first time the comparative validity of these brief cognitive screening instruments for schizophrenia and psychosis (SCIP-S and B-CATS) in relation to a global cognitive score derived from a more detailed assessment battery in patients with a schizophrenia spectrum disorder or type I bipolar disorder.

\section{Methods}

\subsection{Sample description}

Samples were extracted from two original studies, with detailed methods previously reported (Guilera et al., 2009; Pino et al., 2008). The sample reported here was composed of all subjects from either study who had complete data for all cognitive measures of interest, excluding two bipolar patients with highly unusual values (i.e., time to complete the TMT-B more than $6 \mathrm{~min}$ ). The sample consisted of 237 participants, 96 patients with schizophrenia (86 schizophrenia, 9 schizoaffective disorders, and 1 schizophreniform disorder), 65 with type I bipolar disorder, and 76 healthy controls. All subjects provided informed written consent to participate and the studies were approved by the Ethics Committee of the University of Barcelona. Both patient groups were diagnosed according to DSM-IV criteria (American Psychiatric Association, 2000). The control group was interviewed with the Comprehensive Assessment of Symptoms and History (CASH) (Andreasen et al., 1992). The patients who had withdrawn from the previous studies, and were not included in the present analysis, did not differ with respect to socio-demographic

Table 1

Cognitive domains and neuropsychological tasks of BCA, B-CATS, and SCIP.

\begin{tabular}{|c|c|c|c|c|}
\hline \multirow[t]{2}{*}{ Cognitive domain } & \multirow[t]{2}{*}{ Neuropsychological tests } & \multicolumn{3}{|c|}{ Brief cognitive instruments } \\
\hline & & BCA & B-CATS & SCIP \\
\hline \multirow[t]{3}{*}{ Motor and processing speed and attention } & Trail making test - part A & $\mathrm{X}$ & & \\
\hline & Digit symbol coding (WAIS) & & $\mathrm{X}$ & \\
\hline & SCIP processing speed test & & & $\mathrm{X}$ \\
\hline \multirow[t]{3}{*}{ Verbal memory and learning } & Hopkins verbal learning test & $\mathrm{X}$ & & \\
\hline & SCIP verbal learning test - immediate & & & $\mathrm{X}$ \\
\hline & SCIP verbal learning test - delayed & & & $\mathrm{X}$ \\
\hline \multirow[t]{3}{*}{ Fluency } & Letter fluency test & $\mathrm{X}$ & & \\
\hline & Category fluency test & $\mathrm{X}$ & $\mathrm{X}$ & \\
\hline & SCIP letter fluency & & & $\mathrm{X}$ \\
\hline \multirow[t]{2}{*}{ Executive functioning and set-shifting } & Trail making test - part B & $\mathrm{X}$ & $\mathrm{X}$ & \\
\hline & SCIP working memory & & & $\mathrm{X}$ \\
\hline
\end{tabular}

BCA: Brief Cognitive Assessment; B-CATS: Brief Cognitive Assessment Tool for Schizophrenia; SCIP: Screen for Cognitive Impairment in Psychiatry. 
(age, sex, marital status, educational level, and employment status) or clinical characteristics (illness duration, hospital admissions, and mania/depression phases) from the patients remaining in the study, in either the schizophrenia or bipolar subgroup (chi-square and $t$ tests with $p>0.05$ ). Table 2 lists the main socio-demographic and clinical characteristics of the sample. When comparing socio-demographic characteristics between groups, statistically significant differences were found in all comparisons (chi-square and analysis of variance with $p<0.05$ ) except for educational level, while no statistically significant differences were observed between patient groups when comparing clinical variables (illness duration, admissions, and depression symptoms) ( $t$ tests with $p>0.05$ ), except for level of depressive symptomatology $(t(151)=3.811, p<0.05)$.

\subsection{Cognitive assessments}

A full neurocognitive assessment comprising attention, memory, executive function, processing speed, and premorbid intelligence was carried out. The battery included the following thirteen neuropsychological measures: the Wechsler Adult Intelligence Scale-III (Wechsler, 1999) subtests of Arithmetic, Digit Span (total score), Digit Symbol-Coding, Letters and Numbers Sequencing, Symbol Search (total score) and Vocabulary, the Wechsler Memory Scale-III (Wechsler, 2004) subtests of Word list I (total words recalled) and Word list II (total words recalled), the Trail Making Test (Army Individual Test Battery, 1944) parts A and B (time), Semantic Fluency (Estes 1974; Rosen 1980) and categories and percentage of iterative errors on the Wisconsin Card Sorting Test (WCST) (Berg, 1948; Heaton et al., 1993). Additionally, one of the three parallel forms of the SCIP-S was administered to the entire sample. Patient neuropsychological subtest scores were standardized according to the cognitive performance of the healthy control group. Bearing in mind the purpose of the study, three neuropsychological measures were

Table 2

Socio-demographic and clinical descriptives by diagnosis.

\begin{tabular}{|c|c|c|c|c|c|c|}
\hline \multirow[t]{2}{*}{ Variable } & \multicolumn{2}{|c|}{ Schizophrenia } & \multicolumn{2}{|l|}{ Bipolar } & \multicolumn{2}{|c|}{$\begin{array}{l}\text { Healthy } \\
\text { controls }\end{array}$} \\
\hline & Mean & SD & Mean & SD & Mean & SD \\
\hline Age & 37.03 & 8.77 & 41.23 & 10.07 & 37.91 & 8.59 \\
\hline Illness duration (months) & 144.91 & 97.66 & 152.11 & 104.14 & - & - \\
\hline Admissions & 2.58 & 3.80 & 3.31 & 4.49 & - & - \\
\hline HAM-D & 5.35 & 3.23 & 3.44 & 2.77 & - & - \\
\hline PANSS-P & 10.15 & 3.53 & - & - & - & - \\
\hline PANSS-N & 16.81 & 6.33 & - & - & & \\
\hline PANSS-G & 25.78 & 6.94 & - & - & & \\
\hline YMRS & - & - & 1.84 & 2.84 & - & - \\
\hline Mania/depression & - & - & $4.46 / 4.46$ & $3.62 / 4.54$ & - & - \\
\hline Variable & $\mathrm{n}$ & $\%$ & $\mathrm{n}$ & $\%$ & $\mathrm{n}$ & $\%$ \\
\hline \multicolumn{7}{|l|}{ Sex } \\
\hline Males & 70 & 72.9 & 32 & 49.2 & 44 & 57.9 \\
\hline Females & 26 & 27.1 & 33 & 50.08 & 32 & 42.1 \\
\hline \multicolumn{7}{|l|}{ Marital status ${ }^{a}$} \\
\hline Single & 82 & 85.4 & 29 & 44.6 & 17 & 22.4 \\
\hline $\begin{array}{l}\text { Non-single, widow/er, } \\
\text { separated or divorced }\end{array}$ & 14 & 14.6 & 36 & 55.4 & 37 & 48.6 \\
\hline \multicolumn{7}{|l|}{ Educational level } \\
\hline Primary education & 37 & 38.5 & 23 & 35.4 & 25 & 32.9 \\
\hline Secondary education & 40 & 41.7 & 23 & 35.4 & 30 & 39.5 \\
\hline University education & 17 & 17.7 & 18 & 27.7 & 21 & 27.6 \\
\hline Other & 2 & 2.1 & 1 & 1.5 & 0 & 0.0 \\
\hline \multicolumn{7}{|l|}{ Employment status a } \\
\hline Employed (or student) & 27 & 28.1 & 22 & 33.9 & 51 & 67.1 \\
\hline Unemployed (or retired) & 27 & 28.1 & 22 & 33.8 & 4 & 5.3 \\
\hline Disability & 42 & 43.8 & 21 & 32.3 & 0 & 0.0 \\
\hline
\end{tabular}

HAM-D: Hamilton Depression Scale; PANSS: Positive and Negative Syndrome Scale; YMRS: Young Mania Rating Scale.

a Percentages are not $100 \%$ for the healthy controls due to missing data. created: a) the score on the B-CATS, b) the total score on the SCIP-S, and c) a global cognition composite score (GCCS). The score on the B-CATS was computed by averaging the standardized scores of the subtests that compose it (Digit Symbol-Coding, Semantic Fluency, and TMT-B time). The total SCIP-S score is provided directly by the scale, and it was standardized according to the scores obtained by the control group. Finally, the GCCS was obtained by computing the average standardized scores for the remaining ten neuropsychological measures after excluding the three components of the B-CATS.

\subsection{Statistical analysis}

Patient neuropsychological scores were standardized according to the cognitive performance of the healthy control group. The GCCS, B-CATS and SCIP-S scales were subsequently submitted to internal consistency analyses by means of Cronbach's alpha method. The relationship between the two cognitive measures (B-CATS and SCIP-S) and the GCCS was examined with Pearson correlation coefficients. In addition, the B-CATS and SCIP-S were introduced as independent variables in a stepwise regression analysis to quantify the amount of explained variance in GCCS scores within each diagnostic group. Total variance for each model $\left(R^{2}\right)$, and changes in $R^{2}$ resulting from the addition of predictors, were also calculated. Moreover, since both the SCIP-S and B-CATS are brief general cognitive assessment instruments anticipated to produce highly correlated scores, partial and semi-partial correlations were calculated to distinguish shared and unique variance in relation to the GCCS. By squaring both the partial correlation, the resulting $\mathrm{R}^{2}$ determination coefficient can be interpreted in terms of proportion of the GCCS variance explained by a predictor after removing the influence of other predictors on both the GCCS and the predictor. Along the same lines, by squaring the semi-partial correlation, it is possible to infer the unique explained variance of the predictor on the GCCS after removing the influence of other predictors in the criterion variable (Hair et al., 2006). Lastly, multicollinearity that may affect the regression models was evaluated by means of a variance inflation factor (VIF) following the criteria of Kleinbaum et al. (1988), in which VIF $\geq 10$ would suggest collinearity problems.

Additionally, an analysis based on the receiver operating characteristic (ROC) curve was carried out in order to evaluate the ability of the B-CATS and SCIP-S to discriminate between cognitively and noncognitively impaired individuals. Thus, the total sample was binary stratified based on the impairment demonstrated on the GCCS, establishing the cut-off point at $\leq-1$ standard deviation below the normal mean. Several indicators were obtained, i.e., area under the curve (AUC) and its confidence interval, sensitivity and specificity values, positive and negative likelihood ratios (+LR and - LR, respectively), and positive and negative predictive values (PPV and $\mathrm{NPV}$, respectively).

Analyses were performed using SPSS software version 17.0, with a significance level of $\alpha=0.05$.

\section{Results}

Means and standard deviations of B-CATS and SCIP-S total scores, and GCCS for each diagnostic group are shown in Table 3. Schizophrenia and bipolar patients performed between 1 and 1.5 standard deviations lower than the healthy controls on all three cognitive measures.

The reliability analyses demonstrated good internal consistency for GCCS in three comparison groups (Cronbach's alpha of .83, .82, and .81 , respectively for schizophrenia, bipolar, and control healthy groups). Reliabilities were moderate for SCIP-S (alpha of .74, .79, and .67, respectively) and questionable to moderate for B-CATS (alpha of $.55, .60$, and .71 , respectively for schizophrenia, bipolar and control healthy groups).

Table 4 presents the Pearson correlation coefficients between GCCS, B-CATS, and SCIP-S for each diagnostic group. GCCS significantly 
Table 3

Cognitive performance by diagnosis.

\begin{tabular}{|c|c|c|c|c|c|c|}
\hline \multirow{2}{*}{$\begin{array}{l}\text { Cognitive } \\
\text { measure }\end{array}$} & \multicolumn{2}{|c|}{ Schizophrenia } & \multicolumn{2}{|c|}{ Bipolar } & \multicolumn{2}{|c|}{ Healthy controls } \\
\hline & Mean & SD & Mean & SD & Mean & SD \\
\hline GCCS & -1.16 & 0.91 & -1.22 & 0.93 & 0.01 & 0.60 \\
\hline B-CATS & -1.50 & 1.12 & -1.44 & 1.03 & 0.03 & 0.79 \\
\hline SCIP-S & -1.46 & 1.28 & -1.35 & 1.37 & -0.01 & 1.02 \\
\hline
\end{tabular}

GCCS: Global Cognitive Composite Score without B-CATS subtests; B-CATS: Brief Cognitive Assessment Tool for Schizophrenia; SCIP-S: Screen for Cognitive Impairment in Psychiatry - Spanish version.

correlated with both the B-CATS and the SCIP-S, in schizophrenia patients, bipolar patients, and normal healthy controls. Values ranged from .57 (B-CATS and GCCS in type I bipolar disorder) to .76 (SCIP-S and GCCS in schizophrenia). No significant differences were found in Pearson correlation coefficients between the two short forms and the global cognitive score (B-CATS/GCCS versus SCIP-S/GCCS) for schizophrenia, bipolar, and healthy control groups.

The stepwise regression models indicated that both the B-CATS and SCIP-S jointly accounted for between 45 and $65 \%$ of the GCCS variance, with slight differences for each diagnostic group. The SCIP-S was the first significant predictor to enter the three regression equations (Table 5), and alone accounted for 58,38 , and $40 \%$ of the variance in GCCS scores of the schizophrenia, bipolar, and control healthy groups, respectively. Supplemental entry of the B-CATS to the regression model also resulted in significant changes in $\mathrm{R}^{2}$ (i.e., changes of $.08, .09$, and .06 for the three groups, respectively) resulting in global $\mathrm{R}^{2}$ values of $.66, .47$, and .46 .

To account for shared and unique contributions to GCCS variability across diagnostic groups, partial and semi-partial correlations from the regression analyses were examined. Partial correlation represents the correlation between the GCCS and B-CATS after common variance with the SCIP-S has been removed from both the GCCS and the BCATS. In contrast, semi-partial correlation represents the correlation between GCCS and B-CATS once the variance in common with the SCIP-S has been removed from the B-CATS but not from the GCCS. Similarly, the two latter analyses were also applied to the correlations (partial and semi-partial) between the GCCS and SCIP-S. Table 6 shows the partial and semi-partial correlation coefficients between the GCCS and both the B-CATS and the SCIP-S.

The semi-partial correlations indicated that the SCIP-S uniquely accounted for $15 \%, 14 \%$, and $14 \%$ of the variance in GCCS in the schizophrenia, bipolar, and healthy control groups, whereas B-CATS uniquely accounted for $8 \%, 9 \%$, and $6 \%$, respectively, of the variability of the GCCS. No multicollinearity (tolerance and VIF statistics) among measures was found.

The ROC analyses showed that, in the case of B-CATS, the AUC was $.866(.820-.91295 \% \mathrm{CI})$ and for the SCIP-S it was .895 (.849-.940 95\% $\mathrm{CI})$, and both were significantly different from $0.5(p<.05)$. Furthermore, there were no statistically significant differences between areas

Table 4

Pearson correlation coefficients between GCCS, B-CATS and SCIP-S scores.

\begin{tabular}{|c|c|c|c|c|c|c|}
\hline & \multicolumn{3}{|l|}{ B-CATS } & \multicolumn{3}{|c|}{ SCIP-S total score } \\
\hline & Schizophrenia & Bipolar & $\begin{array}{l}\text { Healthy } \\
\text { controls }\end{array}$ & Schizophrenia & Bipolar & $\begin{array}{l}\text { Healthy } \\
\text { controls }\end{array}$ \\
\hline GCCS & .716 & .572 & .573 & .764 & .692 & .658 \\
\hline $\begin{array}{l}\text { SCIP-S } \\
\text { total score }\end{array}$ & .549 & .507 & .568 & - & - & - \\
\hline
\end{tabular}

GCCS: Global Cognitive Composite Score without B-CATS subtests; B-CATS: Brief Cognitive Assessment Tool for Schizophrenia; SCIP-S: Screen for Cognitive Impairment in Psychiatry - Spanish version.

No significant differences were found in Pearson correlation coefficients between the two short forms and the global cognitive score (B-CATS/GCCS versus SCIP-S/GCCS) for schizophrenia, bipolar, and healthy control groups.
Table 5

Stepwise regression analyses with GCCS as criterion and B-CATS and SCIP-S as predictors.

\begin{tabular}{lllll}
\hline Group & Variable & B & Standard error & $t$ test \\
\hline Schizophrenia & SCIP-S & .36 & .06 & $6.37^{* *}$ \\
& B-CATS & .29 & .06 & $4.58^{* *}$ \\
Bipolar & SCIP-S & .27 & .07 & $3.92^{* *}$ \\
& B-CATS & .25 & .08 & $3.09^{*}$ \\
Healthy controls & SCIP-S & .26 & .06 & $4.29^{* *}$ \\
& B-CATS & .22 & .08 & $2.91^{*}$ \\
\hline
\end{tabular}

B-CATS: Brief Cognitive Assessment Tool for Schizophrenia; SCIP-S: Screen for Cognitive Impairment in Psychiatry - Spanish version.

** $p<.001$

$* p<.01$.

under the ROC curve $(z=1.131, p=0.26)$. Table 7 shows the sensitivity and specificity, the likelihood ratios, and the predictive values for most plausible different cut-off points (with a sensitivity value of nearly 80 or more). It is worth remembering that screening tests, such as the B-CATS and SCIP-S, must show high sensitivity and a high NPV in order to minimize the rate of false negatives, even if this leads to a certain increase in the number of false positives. In this sense, both scales show satisfactory results, but there are small differences depending on the cut-off point chosen.

\section{Discussion}

Our results, in this novel research on subjects with a schizophrenia spectrum disorder or type I bipolar disorder, indicate that both the BCATS and the SCIP-S scales are brief cognitive assessment tools with acceptable reliability and concurrent validity. Good to excellent concurrent validity was demonstrated by the good concordance between each of these scales and a GCCS. There were certain discrepancies in severity of impairment between performance in the longer battery and in the brief instruments in the three diagnostic groups and this discrepancy was higher for bipolar than for schizophrenia patients. While the first might suggest a relative excess of 'positives' in patients showing impairment in cognitive performance for the two brief cognitive tests, with regard to the longer battery, this fact would suggest that the two brief instruments probably capture a higher number of 'positives' (cognitive impairment) which, as is common with screening instruments, may to some extent be attributable to false positives. Comparatively, the SCIP-S showed similar performance in schizophrenia, bipolar and healthy control subjects. As expected, the amount of unique variance in the GCCS explained by either the SCIP-S or the B-CATS was lower than the shared or the global variance explained in the regression equations. The SCIP-S contributed a slightly greater amount of unique variance to the GCSS than did the B-CATS in all three diagnostic groups although both have similar correlations with GCSS and similar AUC values in the three diagnostic groups. Slight superiority in alpha coefficients for SCIP regarding B-CATS might in part be due to the fact that SCIP included a higher number of tests than B-CATS. Regarding decision validity, both scales showed good ability to differentiate between individuals with and without cognitive impairment in terms of a global measure of cognitive functioning.

Hurford et al. (2009) demonstrated that the B-CATS is a brief cognitive instrument composed of well-known cognitive subtests with adequate psychometric properties. In fact, it appears to be a good choice for screening cognitive impairment in schizophrenia. The original English version of the SCIP and its Spanish translation (the SCIP-S) show good equivalence of their three alternate forms, good internal consistency, and good test-retest reliability in both healthy control and clinical samples (Pino et al., 2008; Purdon, 2005). In addition, the SCIP-S seems to be a valid instrument for the detection of 
Table 6

Partial and semi-partial correlations between GCCS and both B-CATS and SCIP-S scores.

\begin{tabular}{|c|c|c|c|c|c|c|}
\hline \multirow[t]{2}{*}{ Group } & \multicolumn{2}{|c|}{ Partial correlations (r)/variance explained $\left(\mathrm{R}^{2}\right)$} & \multicolumn{2}{|c|}{ Semi-partial correlations (sr)/variance explained $\left(\mathrm{sr}^{2}\right)$} & \multicolumn{2}{|c|}{ Collinearity } \\
\hline & GCCS and B-CATS & GCCS and SCIP-S & GCCS and B-CATS & GCCS and SCIP-S & Tolerance & VIF \\
\hline Schizophrenia & $.44 / .19$ & $.56 / .31$ & $.28 / .08$ & $.39 / .15$ & .57 & 1.75 \\
\hline Bipolar & $.38 / .14$ & $.46 / .21$ & $.30 / .09$ & $.38 / .14$ & .74 & 1.35 \\
\hline Healthy controls & $.32 / .10$ & $.45 / .20$ & $.25 / .06$ & $.37 / .14$ & .66 & 1.51 \\
\hline
\end{tabular}

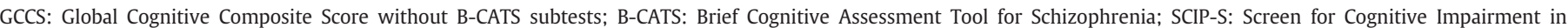
Psychiatry - Spanish version.

cognitive deficits in schizophrenia (Pino et al., 2008; Rojo et al., 2010) and type I bipolar disorder (Guilera et al., 2009).

The SCIP and the B-CATS are pen and pencil instruments, with an average administration time of between 12 and $15 \mathrm{~min}$. They do not require a computer for administration, and they are easily scored manually. Thus, the B-CATS and the SCIP seem to be good candidates as auxiliary assessment tools for the screening of cognitive impairment, and they could provide clinicians with meaningful data regarding their patients' global cognitive function. In contrast to a complete neuropsychological evaluation, professionals from various clinical backgrounds could easily be trained to administer the B-CATS and the SCIP, although supervision by a licensed psychologist is strongly recommended. Both instruments are relatively new, but normative data are available and additional investigations are ongoing to validate the tools for applications to clinical populations. Brief cognitive screening instruments will never replace the diagnostic and monitoring value of a full neuropsychological examination, but they could certainly offer a great deal of value to clinicians working with patients with psychotic disorders, particularly if the diagnostic criteria for schizophrenia are adapted to include reference to quantified cognitive deficits (Keefe, 2008; Barch and Keefe, 2010). Practical considerations of cost and efficiency are important in the selection of an instrument for evaluation of cognitive deficits, particularly given the large number of psychiatric patients (approximately $2 \%$ of the general population) that could benefit from a screening examination. The B-CATS and the SCIP provide reliable and valid results in approximately $15 \mathrm{~min}$. The two tests have some similarities, including somewhat similar tests of visuomotor tracking and verbal fluency, but the SCIP also includes tests of verbal working memory, immediate verbal list learning, and delayed verbal list recall. The B-CATS is made up of elements of other tests, and is thus subject to costs and conditions of the corresponding psychological testing

Table 7

Criterion values and indicators of the ROC curve for B-CATS and SCIP-S.

\begin{tabular}{lllllll}
\hline Criterion & Sensitivity (95\% CI) & Specificity (95\% CI) & +LR & -LR & PPV & NPV \\
\hline$B$-CATS & & & & & & \\
$\leq-1.07$ & $79.07(69.0-87.1)$ & $75.18(67.2-82.1)$ & 3.19 & 0.28 & 66.0 & 85.5 \\
$\leq-1.04$ & $81.40(71.6-89.0)$ & $75.18(67.2-82.1)$ & 3.28 & 0.25 & 66.7 & 86.9 \\
$\leq-1.01$ & $81.40(71.6-89.0)$ & $74.47(66.4-81.4)$ & 3.19 & 0.25 & 66.0 & 86.8 \\
$\leq-1.00$ & $82.56(72.9-89.9)$ & $74.47(66.4-81.4)$ & 3.23 & 0.23 & 66.4 & 87.5 \\
$\leq-0.97$ & $84.88(75.5-91.7)$ & $73.76(65.7-80.8)$ & 3.23 & 0.20 & 66.4 & 88.9 \\
$\leq-0.96$ & $86.05(76.9-92.6)$ & $73.05(64.9-80.2)$ & 3.19 & 0.19 & 66.1 & 89.6 \\
& & & & & & \\
SCIP-S & & & & & & \\
$\leq 68$ & $79.07(69.0-87.1)$ & $84.40(77.3-90.0)$ & 5.07 & 0.25 & 75.6 & 86.9 \\
$\leq 69$ & $81.40(71.6-89.0)$ & $81.56(74.2-87.6)$ & 4.41 & 0.23 & 72.9 & 87.8 \\
$\leq 70$ & $83.72(74.2-90.8)$ & $78.01(70.3-84.5)$ & 3.81 & 0.21 & 69.9 & 88.7 \\
$\leq 71$ & $88.37(79.7-94.3)$ & $75.18(67.2-82.1)$ & 3.56 & 0.15 & 68.5 & 91.4 \\
$\leq 72$ & $89.53(81.1-95.1)$ & $72.34(64.2-79.5)$ & 3.24 & 0.14 & 66.4 & 91.9 \\
$\leq 73$ & $90.70(82.5-95.9)$ & $68.09(59.7-75.7)$ & 2.84 & 0.14 & 63.4 & 92.3 \\
\hline
\end{tabular}

B-CATS: Brief Cognitive Assessment Tool for Schizophrenia; SCIP-S: Screen for Cognitive Impairment in Psychiatry - Spanish version.

CI: confidence interval; + LR: positive likelihood ratio; - LR: negative likelihood ratio; PPV: positive predictive value; NPV: negative predictive value.

a Standardized scores were converted to raw scores because, unlike the B-CATS, the SCIP directly provides a total raw score, which is more easily interpretable. corporations. In contrast, the SCIP is an independent instrument that, although subject to copyright, at the time of publishing this manuscript, can be obtained directly from the authors.

The brief instruments for assessment of cognitive deficits in schizophrenia and other psychosis were not designed for differential diagnosis of cerebral pathology or for the prediction of socio-vocational limitations resulting from cognitive deficits, both of which should be left to professionals with expertise in clinical neuropsychology. However, the brief screening tools appear to have some value for the estimation of global cognitive deficits apparent in psychosis patients. For example, a ROC curve analysis of the sensitivity and the specificity of the SCIP-S supported its use for differentiation between cognitively and noncognitively impaired samples in schizophrenia, bipolar disorder, and healthy controls (Rojo et al., 2010). Further studies of sensitivity and specificity will be necessary for the other cognitive screening tools.

Our results were in keeping with baseline cognitive examination of the large data set comprising 1493 patients with chronic schizophrenia enrolled in the CATIE schizophrenia trial (Keefe, 2008). This study revealed that one of the measures collected in the CATIE battery (WAIS-R digit symbol) accounted for $61 \%$ of the variance in the total score. Moreover, a composite score resulting from the average of digit symbol conjointly with other 5 subtests (list learning, grooved pegboard, letter-number sequencing, verbal fluency, and a maze task) accounted for $93 \%$ of the variance in the global cognitive score (Keefe, 2008).

One limitation of the present study concerns the battery chosen for use as the gold standard. We are aware that it does not explore all the cognitive domains impaired in functional psychoses (e.g., social cognition). Future studies must therefore explore whether B-CATS and SCIP performance could be related to other cognitive domains that are not directly assessed by the test. In addition, SCIP results in psychosis populations should be complemented by ecological measures of current functioning in the community. Kraus and Keefe (2007) rightly suggest that the low face validity of many assessments of cognitive functioning in patients with schizophrenia calls for the co-administration of appropriate measures of functional outcome, such as interview-based and functional proxy measures. It will also be important to further examine the sensitivity of the B-CATS and the SCIP to changes with treatment, particularly in the context of clinical trials assessing cognitive-enhancing properties of novel treatments, and in the assessment of benefits claimed from various cognitive retraining products that are now available or soon to be published. In conclusion, despite the above limitations, the two screening tools offer a relatively simple and inexpensive assessment that could be adapted to mass testing if proven sensitive to change over time. Another limitation of our study concerns the low internal consistency coefficients of B-CATS and SCIP across diagnostic groups. These low alphas should be interpreted in view of several factors: a) the particular test length involved (Cronbach alpha will be higher for longer tests than for shorter tests); b) the context of degree of heterogeneity in performance in each of the groups involved (samples with small variability on the trait will give a smaller alpha than samples with wide variability in traits), and obviously the fact that in the present study, the reliability of each group is analyzed separately which means that the variability decreases; and c) constraints related 
to different measurement errors of tests included within B-CATS and SCIP (Gruijter and van der Kamp, 2008; Streiner, 2003).

In summary, the B-CATS in schizophrenia and the SCIP in schizophrenia and bipolar disorder patients seem to be appropriate tools for brief cognitive assessment in clinical practice, since they reduce the time and resources required for complete neuropsychological assessment and can provide almost as much information as longer batteries for calculating global cognitive performance.

\section{Role of funding source}

This study was funded by Pfizer Spain and supported by project 2009SGR822 of the Departament d'Universitats, Recerca i Societat de la Informació de la Generalitat de Catalunya. This study was also supported by grants from the Ministerio de Sanidad y Consumo, Instituto de Salud Carlos III, RETICS RD06/0011 (REM-TAP Network) and Plan Nacional de Drogas (2008/I/30) and Gobierno de Navarra (GON 55/200). Pfizer Spain participated in the design of the study and engaged a CRO for logistic purposes only. The authors were responsible for data analysis, data interpretation, and manuscript writing for publication. Departament d'Universitats, Recerca i Societat de la Informació de la Generalitat de Catalunya, Ministerio de Educación y Ciencia, Ministerio de Sanidad y Consumo, Instituto de Salud Carlos III, Plan Nacional de Drogas and Gobierno de Navarra had no role other than funding the study.

\section{Contributors}

Manuel Cuesta, Oscar Pino, Georgina Guilera, Emilio Rojo, Juana Gómez were responsible for data analysis, data interpretation, and manuscript writing. Scot E. Purdon provided the SCIP and assisted with translation of the SCIP-S and preparation of this manuscript. Eduard Vieta, Rafael Tabarés-Seisdedos, Anabel Martínez-Arán, Manuel Franco, Benedicto Crespo-Facorro, Nuria Segarra, and Miguel Bernardo were responsible for data interpretation and manuscript writing. Francisco Mesa and Javier Rejas participated in the design of the study and supervision of CRO logistics, data collection, and manuscript drafting. All authors approved the final manuscript.

\section{Conflict of interest}

Javier Rejas and Francisco Mesa are employees of Pfizer Spain. All other authors declare that they have no conflicts of interest.

\section{Acknowledgments}

The authors wish to thank Silvia Martínez (European Biometric Institute, Barcelona, Spain) for her support and help with the logistics of this project.

\section{References}

American Psychiatric Association, 2000. Diagnostic and Statistical Manual of Mental Disorders, Text Revision (DSM-IV TR), Fourth Edition. . Washington DC.

Andreasen, N.C., Flaum, M., Arndt, S., 1992. The Comprehensive Assessment of Symptoms and History (CASH). An instrument for assessing diagnosis and psychopathology. Arch. Gen. Psychiatry 49, 615-623.

Army Individual Test Battery, 1944. Manual of Directions and Scoring. War Department, Adjutant General's Office, Washington, DC.

Barch, D.M., Keefe, R.S., 2010. Anticipating DSM-V: opportunities and challenges for cognition and psychosis. Schizophr Bull. 36, 43-47.

Berg, E.A., 1948. Sample objective test for measuring flexibility and thinking. J. Gen. Psychol. 39, 15-22.

Brewer, W.J., Francey, S.M., Wood, S.J., et al., 2005. Memory impairments identified in people at ultra-high risk for psychosis who later develop first-episode psychosis. Am. J. Psychiatry 162, 71-78.

Daban, C., Martinez-Aran, A., Torrent, C., et al., 2006. Specificity of cognitive deficits in bipolar disorder versus schizophrenia. A systematic review. Psychother. Psychosom. 75, 72-84.

Dominguez, M. de G., Viechtbauer, W., Simons, C.J., et al., 2009. Are psychotic psychopathology and neurocognition orthogonal? A systematic review of their associations. Psychol. Bull. 135, 157-171.

Estes, W.K., 1974. Learning theory and intelligence. Am. Psychol. 29, 740-749.

Folstein, M.F., Folstein, S.E., McHugh, P.R., 1975. Mini-mental state: a practical method for grading the cognitive state of patients for the clinician. J. Psychiatr. Res. 12, 189-198.

Green, M.F., Kern, R.S., Heaton, P.K., 2004. Longitudinal studies of cognition and functional outcome in schizophrenia: implications for MATRICS. Schizophr. Res. 72, 41-51.

Green, M.F., Olivier, B., Crawley, J.N., et al., 2005. Social cognition in schizophrenia: recommendations from the measurement and treatment research to improve cognition in schizophrenia new approaches conference. Schizophr. Bull. 31, 882-887.

Gruijter, D.N.M., van der Kamp, L.J.Th., 2008. Statistical Test Theory for the Behaviora Sciences. Chapman \& Hall/CRC, Boca Raton, FL.

Guilera, G., Pino, O., Gómez-Benito, J., et al., 2009. Clinical usefulness of the Screen for Cognitive Impairment in Psychiatry (SCIP-S) scale in patients with type I bipolar disorder. HQLO 7, 28.

Hair Jr., J.F., Black, W.C., Babin, B.J., et al., 2006. Multivariate Data Analysis, 6th ed. Pearson Prentice Hall, Upper Saddle River, NJ.

Heaton, R.K., Chelune, G.J., Talley, J.L., et al., 1993. Wisconsin Card Sorting Test (WCST) Manual-Revised and Expanded. Psychological Assessment Resources, Odessa, FL

Heinrichs, R.W., Zakzanis, K.K., 1998. Neurocognitive deficit in schizophrenia: a quantitative review of the evidence. Neuropsychology 12, 426-445.

Hurford, I.M., Marder, S.R., Keefe, R.S., et al., 2009. A brief cognitive assessment tool for schizophrenia: construction of a tool for clinicians. Schizophr. Bull. doi:10.1093/ schbul/sbp095

Keefe, R.S., 2008. Should cognitive impairment be included in the diagnostic criteria for schizophrenia? World Psychiatry 7, 22-28.

Keefe, R.S.E., Goldberg, T.E., Harvey, P.D., 2004. The Brief Assessment of Cognition in Schizophrenia: reliability, sensitivity, and comparison with a standard neurocognitive battery. Schizophr. Res. 68, 283-297.

Kiernan, R.J., Mueller, J., Langston, J.W., et al., 1987. The neurobehavioral cognitive status examination: a brief but quantitative approach to cognitive assessment. Ann. Intern. Med. 107, 481-485.

Kleinbaum, D.G., Kupper, L.L., Muller, K.E., 1988. Applied Regression Analysis and Other Multivariables Methods. PWS-KENT, Boston.

Krabbendam, L., Arts, B., van Os, J., et al., 2005. Cognitive functioning in patients with schizophrenia and bipolar disorder: a quantitative review. Schizophr. Res. 80 137-149.

Kraus, M.S., Keefe, R.S.E., 2007. Cognition as an outcome measure in schizophrenia. Br. J. Psychiatry 191, 46-51.

Nieuwenstein, M.R., Aleman, A., de Haan, E.H., 2001. Relationship between symptom dimensions and neurocognitive functioning in schizophrenia: a meta-analysis of WCST and CPT studies. Wisconsin Card Sorting Test. Continuous Performance Test. J. Psychiatr. Res. 35, 119-125.

Pino, O., Guilera, G., Rojo, E., et al., 2008. Spanish version of the Screen for Cognitive Impairment in Psychiatry (SCIP-S): Psychometric properties of a brief scale for cognitive evaluation in schizophrenia. Schizophr. Res. 99, 139-148.

Purdon, S.E., 2005. The Screen for Cognitive Impairment in Psychiatry (SCIP): instructions and three alternate forms. PNL Inc, Edmonton, Alberta.

Randolph, C., Tierney, M.C., Mohr, E., et al., 1998. The Repeatable Battery for the Assessment of Neuropsychological Status (RBANS): preliminary clinical validity. J. Clin. Exp. Neuropsychol. 20, 310-319.

Reichenberg, A., Harvey, P.D., Bowie, C.R., et al., 2009. Neuropsychological function and dysfunction in schizophrenia and psychotic affective disorders. Schizophr. Bull. 35 1022-1029.

Rojo, E., Pino, O., Guilera, G., et al., 2010. Neurocognitive diagnosis and cut-off scores of the Screen for Cognitive Impairment in Psychiatry (SCIP-S). Schizophr. Res. 116, $243-251$.

Rosen, W.G., 1980. Verbal fluency in aging and dementia. J. Clin. Neuropsychol. 2, 135-146.

Segarra, N., Bernardo, M., Gutierrez, F., et al., 2010. Spanish validation of the Brief Assessment in Cognition in Schizophrenia (BACS) in patients with schizophrenia and healthy controls. Eur. Psychiatry. doi:10.1016/j.eurpsy.2009.11.001.

Seidman, L.J., Giuliano, A.J., Meyer, E.C., et al., 2010. Neuropsychology of the prodrome to psychosis in the NAPLS consortium: relationship to family history and conversion to psychosis. Arch. Gen. Psychiatry 67, 578-588.

Streiner, D.L., 2003. Starting at the beginning: an introduction to coefficient alpha and internal consistency. J. Pers. Assess. 80, 99-103.

Tabarés-Seisdedos, R., Balanzá-Martínez, V., Sánchez-Moreno, J., et al., 2008. Neurocognitive and clinical predictors of functional outcome in patients with schizophrenia and bipolar I disorder at one-year follow-up. J. Affect. Disord. 109, 286-299.

Toulopoulou, T., Picchioni, M., Rijsdijk, F., et al., 2007. Substantial genetic overlap between neurocognition and schizophrenia: genetic modelling in twin samples. Arch. Gen. Psychiatry 64, 1348-1355.

Velligan, D.I., DiCocco, M., Bow-Thomas, C.C., et al., 2004. A brief cognitive assessment for use with schizophrenia patients in community clinics. Schizophr. Res. 71 273-783.

Ventura, J., Nuechterlein, K.H., Subotnik, K.L., et al., 2000. Symptom dimensions in recent-onset schizophrenia and mania: a principal components analysis of the 24 item Brief Psychiatric Rating Scale. Psychiatry Res. 97, 129-135.

Wechsler, D., 1999. Wechsler Adults Intelligence Scale 3, WAIS-III Manual. Spanish version. TEA Ediciones, Madrid.

Wechsler, D., 2004. Wechsler Memory Scale 3, WMS-III Manual. Spanish version. TEA Ediciones, Madrid.

Woodcock, R.W., McGrew, K.S., Mather, N., 2001. Woodcock-Johnson III Tests of Cognitive Abilities. Riverside, Itasca, IL. 